\title{
Influence of magnetic field on the peristaltic flow of a viscous fluid through a finite-length cylindrical tube
}

\author{
S.K. Pandey and Dharmendra Tripathi* \\ Department of Applied Mathematics, Institute of Technology, Banaras Hindu University, Varanasi 221005, India
}

(Received 10 June 2009; final version received 29 January 2010)

\begin{abstract}
The paper presents an analytical investigation of the peristaltic transport of a viscous fluid under the influence of a magnetic field through a tube of finite length in a dimensionless form. The expressions of pressure gradient, volume flow rate, average volume flow rate and local wall shear stress have been obtained. The effects of the transverse magnetic field and electrical conductivity (i.e. the Hartmann number) on the mechanical efficiency of a peristaltic pump have also been studied. The reflux phenomenon is also investigated. It is concluded, on the basis of the pressure distribution along the tubular length and pumping efficiency, that if the transverse magnetic field and the electric conductivity increase, the pumping machinery exerts more pressure for pushing the fluid forward. There is a linear relation between the averaged flow rate and the pressure applied across one wavelength that can restrain the flow due to peristalsis. It is found that there is a particular value of the averaged flow rate corresponding to a particular pressure that does not depend on the Hartmann number. Naming these values 'critical values', it is concluded that the pressure required for checking the flow increases with the Hartmann number above the critical value and decreases with it below the critical value. It is also inferred that magneto-hydrodynamic parameters make the fluid more prone to flow reversal. The conclusion applied to oesophageal swallowing reveals that normal water is easier to swallow than saline water. The latter is more prone to flow reversal. A significant difference between the propagation of the integral and non-integral number of waves along the tube is that pressure peaks are identical in the former and different in the latter cases.
\end{abstract}

Keywords: peristaltic flow; magneto-hydrodynamics; finite-length tube; mechanical efficiency; reflux

\section{Introduction}

Physiological fluids are transported from one place to another by continuous muscle contractions and relaxations. This type of transportation is called peristaltic transport. The flow of blood through arteries and veins, the passage of urine through ureters, the flow of bile from the gall bladder into the duodenum, the movement of chyme in the entire gastro-intestinal tract, the transportation of food boluses through the alimentary canal and the movement of some worms are some important examples of peristaltic transport.

Magneto-hydrodynamics is the study of dynamics of magnetic field in electrically conducting fluids. Examples of such fluids include plasma (blood), liquid metals and salt or sea water. The idea of magneto-hydrodynamics is that magnetic fields can induce currents in a moving conducting fluid, which create forces on the fluid, and also change the magnetic field itself. The equations describing magneto-hydrodynamic (MHD) fluids are a combination of Navier-Stokes equations and Maxwell equations of electromagnetism.

Recently, several investigators (El-Shehawey and Husseny 2002; Mekheimer 2004; Hayat and Ali 2006, 2007, 2008; Naby et al. 2006a, 2006b; Hayat et al. 2007a, 2007b, 2008a, 2008b; Ali et al. 2008; Ebaid 2008; Kothandapani and Srinivas 2008a, 2008b; Mekheimer 2008; Mekheimer and Abd-elmaboud 2008; Wang et al. 2008) have studied the effects of magnetic field on the peristaltic flow of physiological fluids. Most of them have studied the effects of the Hartmann number on the pressure and the friction forces on steady flows in infinite channels or tubes. The result is that pressure increases as the Hartmann number increases whether the wall motions are symmetric or asymmetric. A few authors (Ali et al. 2008; Mekheimer 2008; Mekheimer and Abd-elmaboud 2008; Kothandapani and Srinivas 2008a, 2008b) have examined the effects of the Hartmann number on the fluid trapped. They found that the size of the trapped bolus decreases as the Hartmann number increases (Ali et al. 2008).

However, none of the researchers has shown an interest in the reflux and mechanical efficiency in a tube. Another important fact is that in physiological flows, the vessels contract and relax but do not expand so that the wall equations to model such flows will definitely differ from the ones fluctuating about the boundary line. Several physiological fluids, such as blood, behave like an MHD fluid.

*Corresponding author: Email: dtripathi.rs.apm@itbhu.ac.in 


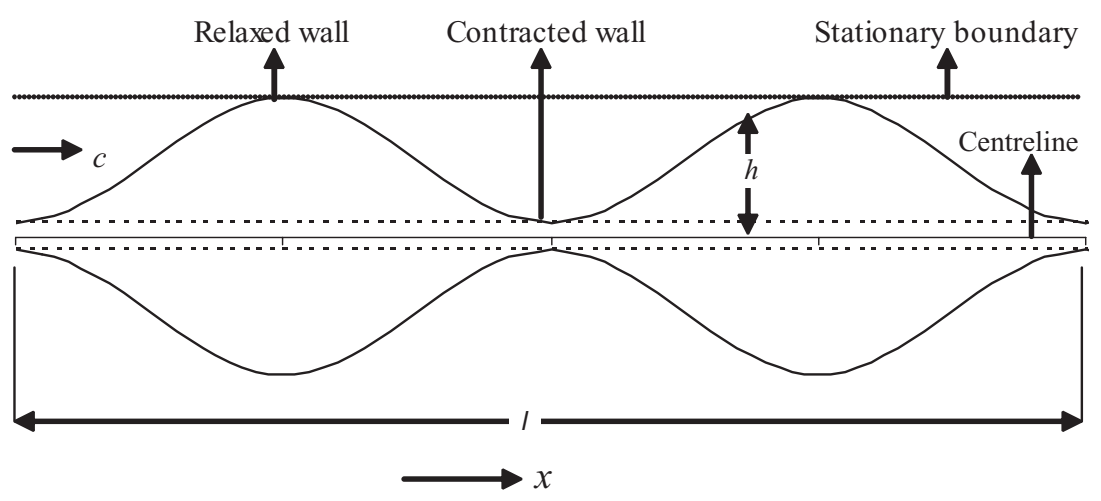

Figure 1. The diagram, based on Equation (1), represents the propagation of a progressive transverse wave along the walls of the tube containing fluid, which undergoes contraction and relaxation but no expansion beyond the boundary. Here $h$ is the radial displacement of the wall, $c$ is the wave velocity, $l$ is the length of the tube and $x$ is the axial distance.

Li and Brausseur (1993) studied the peristaltic transport of a food bolus of the Newtonian type through the oesophagus by considering a finite-length tube, which was followed by Misra and Pandey (2001), who considered power-law fluids.

We intend to investigate the peristaltic transport of an MHD fluid in a cylindrical tube of finite length under the influence of progressive contraction waves that contract, relax but do not expand and propagate along its walls. The model is applicable to swallowing of conducting fluids, such as saline water, through the oesophagus and blood flow through the aorta. Both the vessels are small in length. This model will fit an artificial peristaltic pump carrying a saline solution for engineering applications.

\section{Mathematical model}

The wall motion is supposed to be created by waves propagating unidirectionally along the tube walls (cf. Figure 1), which are mathematically modelled as

$$
\tilde{h}(\tilde{x}, \tilde{t})=a-\tilde{\phi} \cos ^{2} \frac{\pi}{\lambda}(\tilde{x}-c \tilde{t})
$$

where $\tilde{h}, \tilde{x}, \tilde{t}, a, \tilde{\phi}, \lambda$ and $c$ represent the radial distance of the wall from the centreline, axial coordinate, time, radius of the tube, amplitude of the wave, wavelength and wave velocity, respectively. It is assumed that as soon as the waves reach the other end of the tube, they die out.

We consider the flow of an incompressible MHD fluid under the effect of the transverse magnetic field $\mathbf{B}=\left(0, B_{0}, 0\right)$, vibrating in a small tube. The walls of the tube are assumed to be electrically insulated, and the induced magnetic field is assumed to be negligible. The equations governing the motion of MHD fluid in the laboratory frame are

$$
\left.\begin{array}{l}
\rho\left(\frac{\partial}{\partial \tilde{t}}+\tilde{u} \frac{\partial}{\partial \tilde{x}}+\tilde{v} \frac{\partial}{\partial \tilde{r}}\right) \tilde{u} \\
=-\frac{\partial \tilde{p}}{\partial \tilde{x}}+\mu\left\{\frac{1}{\tilde{r}} \frac{\partial}{\partial \tilde{r}}\left(\tilde{r} \frac{\partial \tilde{u}}{\partial \tilde{r}}\right)+\frac{\partial^{2} \tilde{u}}{\partial \tilde{x}^{2}}\right\}-\sigma B_{0}^{2} \tilde{u}, \\
\rho\left(\frac{\partial}{\partial \tilde{t}}+\tilde{u} \frac{\partial}{\partial \tilde{x}}+\tilde{v} \frac{\partial}{\partial \tilde{r}}\right) \tilde{v} \\
=-\frac{\partial \tilde{p}}{\partial \tilde{r}}+\mu\left\{\frac{\partial}{\partial \tilde{r}}\left(\frac{1}{\tilde{r}} \frac{\partial}{\partial \tilde{r}}(\tilde{r} \tilde{v})\right)+\frac{\partial^{2} \tilde{v}}{\partial \tilde{x}^{2}}\right\}, \\
\frac{\partial \tilde{u}}{\partial \tilde{x}}+\frac{1}{r} \frac{\partial(\tilde{r} \tilde{v})}{\partial \tilde{r}}=0 .
\end{array}\right\}
$$

We introduce the following non-dimensional parameters:

$$
\begin{aligned}
& x=\frac{\tilde{x}}{\lambda}, \quad r=\frac{\tilde{r}}{a}, \quad t=\frac{c \tilde{t}}{\lambda}, \quad t_{m}=\frac{c \tilde{t}_{m}}{\lambda}, \quad u=\frac{\tilde{u}}{c}, \\
& v=\frac{\tilde{v}}{c \alpha}, \quad \alpha=\frac{a}{\lambda}, \quad h=\frac{\tilde{h}}{a}, \quad l=\frac{\tilde{l}}{\lambda}, \\
& \phi=\frac{\tilde{\phi}}{a}, p=\frac{\tilde{a} a^{2}}{\mu c \lambda}, \psi=\frac{\tilde{\psi}}{\pi a^{2} c}, Q=\frac{\tilde{Q}}{\pi a^{2} c}, \\
& R e=\frac{\rho c a \alpha}{\mu}, \quad M=\sqrt{\frac{\sigma}{\mu}} a B_{0},
\end{aligned}
$$

where $r, u, v, \alpha, p, \psi, Q$ and $\rho, R e, M, \sigma, B_{0}$ denote the dimensionless counterparts of radial coordinate, axial velocity, radial velocity, wave number, pressure, streamfunction, volume flow rate and fluid density, Reynolds number, Hartmann number, electrical conductivity and strength of the magnetic field, respectively. Applying the long wavelength and small-Reynolds-number approximation, Equations (2) reduce to

$$
\left.\begin{array}{l}
\frac{\partial p}{\partial x}=\frac{1}{r} \frac{\partial}{\partial r}\left(r \frac{\partial u}{\partial r}\right)-M^{2} u, \\
\frac{\partial p}{\partial r}=0, \\
\frac{\partial u}{\partial x}+\frac{1}{r} \frac{\partial(r v)}{\partial r}=0 .
\end{array}\right\}
$$


Imposing the boundary conditions

$$
\left.\begin{array}{l}
u(x, h, t)=0, v(r, h, t)=\frac{\partial h}{\partial t} \\
\frac{\partial u(x, 0, t)}{\partial r}=\text { finite, } v(r, 0, t)=0
\end{array}\right\}
$$

on the governing Equations (4), the axial and radial velocities are obtained as

$$
\begin{gathered}
u=\frac{1}{M^{2}} \frac{\partial p}{\partial x}\left(\frac{I_{0}(M r)}{I_{0}(M h)}-1\right), \\
v=\frac{1}{M^{2}}\left[\frac{\partial p}{\partial x} \frac{\partial h}{\partial x}\left\{\frac{I_{1}(M r) I_{1}(M h)}{\left(I_{0}(M h)\right)^{2}}\right\}\right. \\
\left.-\frac{\partial^{2} p}{\partial x^{2}}\left\{\frac{I_{1}(M r)}{M I_{0}(M h)}-\frac{r}{2}\right\}\right],
\end{gathered}
$$

where $I_{0}$ and $I_{1}$ are the modified Bessel functions of the zero and the first order of the first kind, respectively.

The transverse velocity, at the boundary of wall, is obtained by substituting the third condition of Equation (5) into Equation (7). It yields

$$
\begin{aligned}
h \frac{\partial h}{\partial t}= & \frac{1}{M^{2}}\left[\frac{\partial p}{\partial x} \frac{\partial h}{\partial x} \frac{h\left(I_{1}(M h)\right)^{2}}{\left(I_{0}(M h)\right)^{2}}\right. \\
& \left.-\frac{\partial^{2} p}{\partial x^{2}}\left(\frac{h I_{1}(M h)}{M I_{0}(M h)}-\frac{h^{2}}{2}\right)\right] .
\end{aligned}
$$

Integrating Equation (8) once with respect to $x$, the pressure gradient is obtained as

$$
\frac{\partial p}{\partial x}=\frac{2 M^{3}}{h}\left[\frac{G(t)+\int_{0}^{x} h \frac{\partial h}{\partial t} d s}{M h-\frac{2 I_{1}(M h)}{I_{0}(M h)}}\right]
$$

where $G(t)$ is an arbitrary function of $t$.

Furthermore, integrating Equation (9) from 0 to $x$, the pressure difference is obtained as

$$
p(x, t)-p(0, t)=2 M^{3} \int_{0}^{x} \frac{1}{h}\left[\frac{G(t)+\int_{0}^{s} h \frac{\partial h}{\partial t} d s_{1}}{M h-\frac{2 I_{1}(M h)}{I_{0}(M h)}}\right] d s .
$$

The substitution $x=l$, in Equation (10), readily gives the pressure difference between the inlet and outlet of the tube as

$$
p(l, t)-p(0, t)=2 M^{3} \int_{0}^{l} \frac{1}{h}\left[\frac{G(t)+\int_{0}^{x} h \frac{\partial h}{\partial t} d s}{M h-\frac{2 I_{1}(M h)}{I_{0}(M h)}}\right] d x,
$$

from which $G(t)$ is determined as

$$
G(t)=\frac{\frac{p(l, t)-p(0, t)}{2 M^{3}}-\int_{0}^{l} \frac{\int_{0}^{x} h \frac{\partial h}{\partial t} d s}{h\left(M h-\frac{2 I_{1}(M h)}{I_{0}(M h)}\right)} d x}{\int_{0}^{l} \frac{1}{h\left(M h-\frac{2 I_{1}(M h)}{I_{0}(M h)}\right)} d x}
$$

The instantaneous volume flow rate in the laboratory frame is defined as $Q(x, t)=\int_{0}^{h} 2 r u d r$, which, by virtue of Equation (6), is given as

$$
Q(x, t)=\frac{1}{M^{2}} \frac{\partial p}{\partial x}\left\{\frac{2 h I_{1}(M h)}{M I_{0}(M h)}-h^{2}\right\} .
$$

However, the following relations exist between the averaged flow rate $\bar{Q}$ and the flow rate $q$ in the wave frame and also the flow rate $Q$ in the laboratory frame:

$$
\bar{Q}=q+1-\phi+\frac{3 \phi^{2}}{8}=Q-h^{2}+1-\phi+\frac{3 \phi^{2}}{8} \text {. }
$$

A simple manipulation of Equation (13), followed by an application of Equation (14), yields the pressure gradient as

$$
\frac{\partial p}{\partial x}=\frac{M^{2}\left(\bar{Q}+h^{2}-1+\phi-\frac{3 \phi^{2}}{8}\right)}{\left\{\frac{2 h I_{1}(M h)}{M I_{0}(M h)}-h^{2}\right\}},
$$

which, on integration between 0 and $x$, gives

$$
p(x)-p(0)=M^{2} \int_{0}^{x}\left[\frac{\bar{Q}+h^{2}-1+\phi-\frac{3 \phi^{2}}{8}}{\left\{\frac{2 h I_{1}(M h)}{M I_{0}(M h)}-h^{2}\right\}}\right] d s .
$$

For $x=l$, it gives the pressure difference between the inlet and the outlet of the tube as

$$
p(l)-p(0)=M^{2} \int_{0}^{l}\left[\frac{\bar{Q}+h^{2}-1+\phi-\frac{3 \phi^{2}}{8}}{\left\{\frac{2 h I_{1}(M h)}{M I_{0}(M h)}-h^{2}\right\}}\right] d x .
$$

The local wall shear stress defined as $\tau_{w}=$ $\left.(\partial u / \partial r)\right|_{r=h}$, reduces, by virtue of Equations (6) and (9), to

$$
\tau_{w}=\frac{2 M^{2}}{h}\left[\frac{G(t)+\int_{0}^{x} h \frac{\partial h}{\partial t} d s}{\frac{M h I_{0}(M h)}{I_{1}(M h)}-2}\right] .
$$




\section{Mechanical efficiency}

It is the ratio of the average rate per wavelength at which work is done by the moving fluid against a pressure head and the average rate at which the walls do work on the fluid (cf. Shapiro et al. 1969). It is derived as

$$
E=\frac{\bar{Q} \Delta p_{1}}{2 \phi\left[I_{11}+\phi I_{12}-(1+\phi) \Delta p_{1}\right]},
$$

where $\Delta p_{1}$ is the pressure difference for one wavelength, i.e. $p(1)-p(0)$ and given from Equation (16) in the form

$$
\begin{aligned}
p(1)-p(0) & =M^{2} \int_{0}^{1}\left[\frac{\bar{Q}+h^{2}-1+\phi-\frac{3 \phi^{2}}{8}}{\left\{\frac{2 h I_{1}(M h)}{M I_{0}(M h)}-h^{2}\right\}}\right] d x \\
I_{11} & =\int_{0}^{1} \frac{\partial p}{\partial x} \cos (2 \pi x) d x \\
I_{12} & =\int_{0}^{1} \frac{\partial p}{\partial x} \cos ^{4}(\pi x) d x
\end{aligned}
$$

and the maximum averaged flow rate, obtained by taking $\Delta p_{1}=0$ in Equation (20), is

$$
\bar{Q}_{0}=1-\phi+\frac{3 \phi^{2}}{8}-\frac{\int_{0}^{1} \frac{h^{2}}{\left\{\frac{2 h I_{1}(M h)}{M I_{0}(M h)}-h^{2}\right\}} d x}{\int_{0}^{1} \frac{1}{\left\{\frac{2 h 1_{1}(M h)}{M l_{0}(M h)}-h^{2}\right\}} d x} .
$$

\section{Reflux limit}

Reflux is an inherent phenomenon of peristaltic movement, which refers to the presence of fluid particles that move, on the average, in a direction opposite to the net flow (cf. Shapiro et al. 1969).

The dimensional form of the streamfunction in the wave frame is defined as

$$
d \tilde{\psi}=2 \pi \tilde{R}(\tilde{U} d \tilde{R}-\tilde{V} d \tilde{X}),
$$

where $\tilde{\psi}, \tilde{R}, \tilde{X}, \tilde{U}$ and $\tilde{V}$ are the streamfunction, coordinates and velocity components, respectively. We use the transformations between the wave and the laboratory frame defined as

$$
\begin{aligned}
\tilde{X} & =\tilde{x}-c \tilde{t}, \quad \tilde{R}=\tilde{r}, \quad \tilde{U}=\tilde{u}-c, \quad \tilde{V}=\tilde{v} \\
\tilde{q} & =\tilde{Q}-c \tilde{h}^{2}, \quad \tilde{\Psi}=\tilde{\psi}-\tilde{r}^{2}
\end{aligned}
$$

where the parameters on the left-hand side are in the wave frame and those on the right-hand side are in the laboratory frame. We obtain the streamfunction by solving Equations
(6), (7), (24) and (25) in the form

$$
\psi=\left\{\frac{\bar{Q}-1+\phi-\frac{3 \phi^{2}}{8}+h^{2}}{\frac{2 h I_{1}(M h)}{M I_{0}(M h)}-h^{2}}\right\}\left\{\frac{2 r I_{1}(M r)}{M I_{0}(M h)}-r^{2}\right\}-r^{2} .
$$

At the wall

$$
\left.\psi\right|_{r=h}=\psi_{w}=\bar{Q}-1+\phi-\frac{3 \phi^{2}}{8} .
$$

The reflux flow rate $Q_{\psi}(x)$ is defined as

$$
Q_{\psi}(x)=\psi+r^{2}(\psi, x) .
$$

Averaging the above equation for one cycle, we get

$$
\bar{Q}_{\psi}=\psi+\int_{0}^{1} r^{2}(\psi, x) d x .
$$

In order to evaluate the above integration, using the perturbation method, $r^{2}(\psi, x)$ is expanded in a power series in terms of a small parameters $\varepsilon$ about the wall as

$$
r^{2}(\psi, x)=h^{2}+a_{1} \varepsilon+a_{2} \varepsilon^{2}+\cdots .
$$

Solving Equations (26) and (27), for a small value of $M$ and comparing the coefficient of $\varepsilon, \varepsilon^{2}, \ldots$, we get

$$
a_{1}=-1, a_{2}=-\frac{M^{2}}{8}\left[\frac{\left(\bar{Q}+h^{2}-1+\phi-\frac{3 \phi^{2}}{8}\right)}{h^{2}-\frac{2 h I_{1}(M h)}{M I_{0}(M h)}}\right] \text {. }
$$

Reflux occurs when the reflux condition, i.e. $\left(\bar{Q}_{\psi} / \bar{Q}\right)>$ 1 , as $\varepsilon \rightarrow 0$ satisfies. Therefore, the reflux limit is obtained by using Equations (29)-(31) as

$$
\bar{Q}<1-\phi+\frac{3 \phi^{2}}{8}-\frac{\int_{0}^{1} \frac{h^{2}}{h^{2}-\frac{2 h 1_{1}(M h)}{M I_{0}(M h)}} d x}{\int_{0}^{1} \frac{1}{h^{2}-\frac{2 h 1_{1}(M h)}{M I_{0}(M h)}} d x} .
$$

\section{Numerical results and discussion}

We now investigate temporal influence and the effect of the Hartmann number, $M$, on the flow behaviour. To this end, we consider the fluid in a tube trapped within a train of two waves propagating along the walls at a time. The peristaltic characteristics can be precisely understood if the pressures at the two ends of the tube are considered to be zero. This can be achieved by a straightforward substitution $p(l, t)-p(0, t)=0$ in Equation (12), which simplifies Equation (10) to a great extent. Graphs based on the 


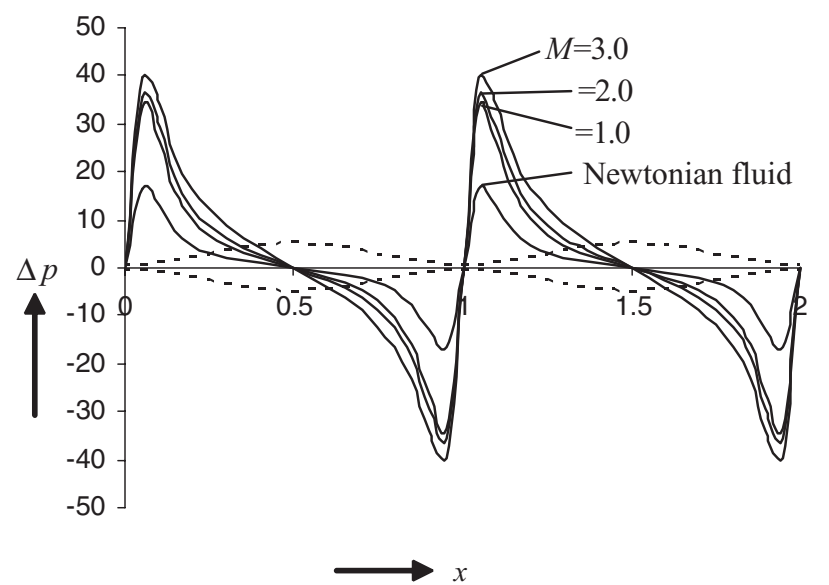

(a) $t=0.0$ (similar is the distribution for $t=1.0$ )

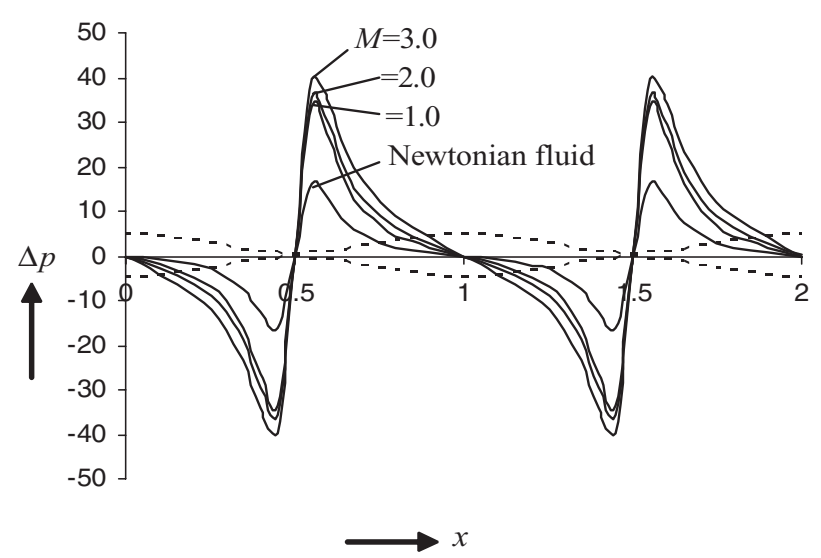

(c) $t=0.5$

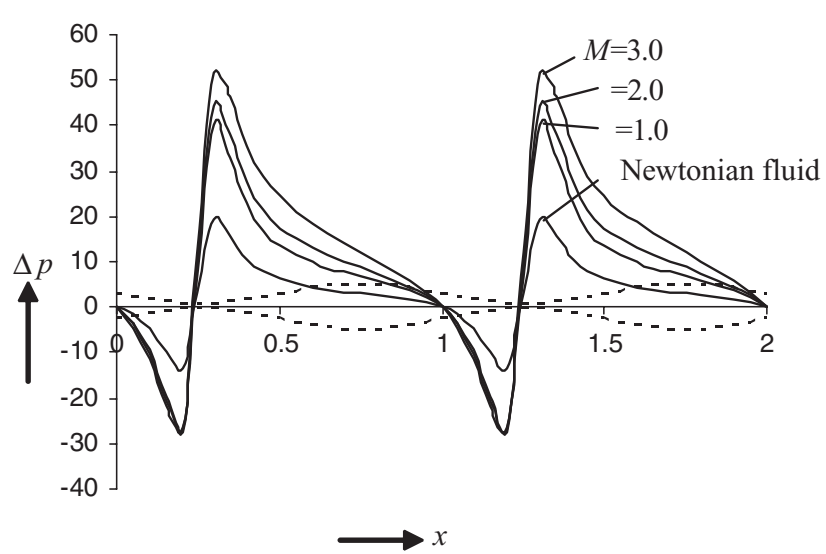

(b) $t=25.0$

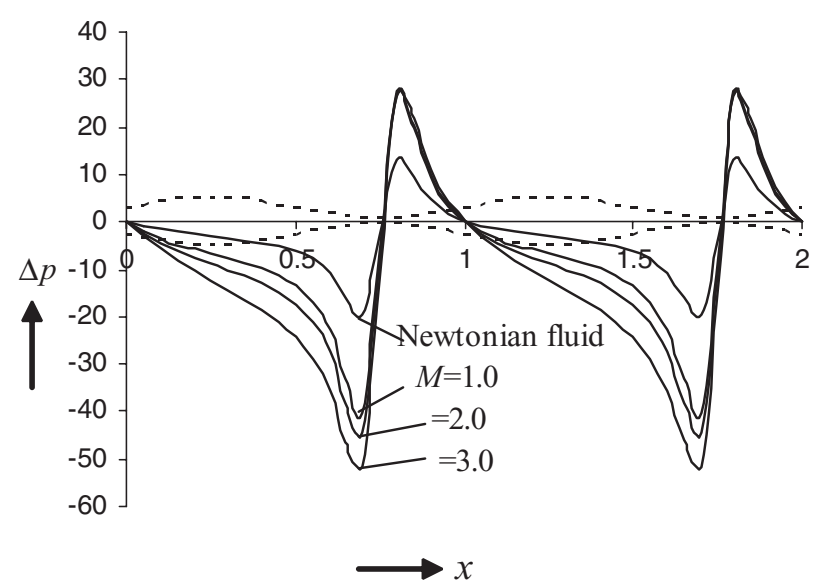

(d) $t=0.75$

Figure 2. Pressure vs. axial distance. Dotted lines represent the position of bolus and solid lines show the pressure distribution, based on Equation (10), across the length of the tube passing the Newtonian and MHD fluids for different Hartmann numbers $(M=1.0,2.0,3.0)$, $\phi=0.9$ and $l=2.0$.

simplified Equation (10) between the tube length and the positional pressure indicate the following facts.

The pressure distribution along the tube length favours the flow, which is clear from the diagrams of Figure 2 at $t=0.0-1.0$. Furthermore, in the first half of the temporal cycle $(t=0.0-0.5)$, there is a tendency for the pressure to take positive values, while in the latter half $(t=0.5-$ 1.0.) the pressure takes negative values. This effect may be attributed to activities taking place at the inlet and the outlet, where the fluid is pulled and pushed, respectively.

Having kept the other parameters constant, if the Hartmann number $M$ is increased, the pressure difference is found to rise in magnitude all along the tube to push the fluid content. As $M \rightarrow 0$, the fluid turns Newtonian. This can, on the other hand, be interpreted that the pumping has to be more efficient. Thus, the fact that $M=\sqrt{(\sigma / \mu)} a B_{0}$ is a function of $B_{0}$ and $\sigma$ reveals that for higher values of the transverse magnetic field $B_{0}$ and the electric conductivity $\sigma$, the pump has to exert more pressure to move the same amount of fluid; however, the effect of the latter is less and it precisely varies as its square root. Since the expression is implicit, an exact variation cannot be given.

In Figure 3, we have considered the propagation of a non-integral number of waves $(l=1.8)$ in the train, which is an inherent characteristics of finite-length tubes. Some significant differences are observed between the integral and non-integral number of waves present in the train propagating along the container wall. Figure 3 shows the temporal effects on the pressure distribution along the tubular length under magnetic field. The more is the symmetry of distribution of boluses in the tube, the less is the difference between the pressure peaks (cf. Figure 3c). The effects of the Hartmann number, $M$, on the pressure distribution for the two distinct wave propagations (integral and non-integral) 


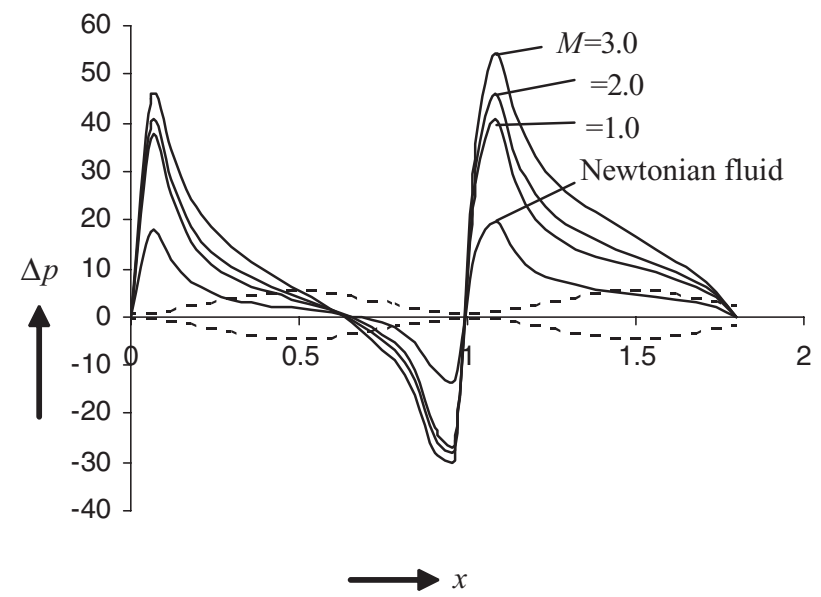

(a) $t=0.0$ (similar is the distribution for $t=1.0$ )

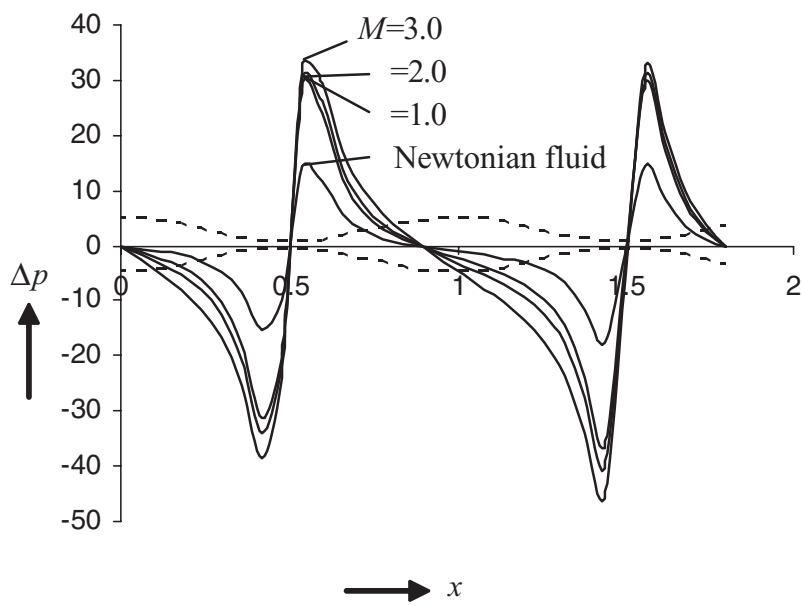

(c) $t=0.5$

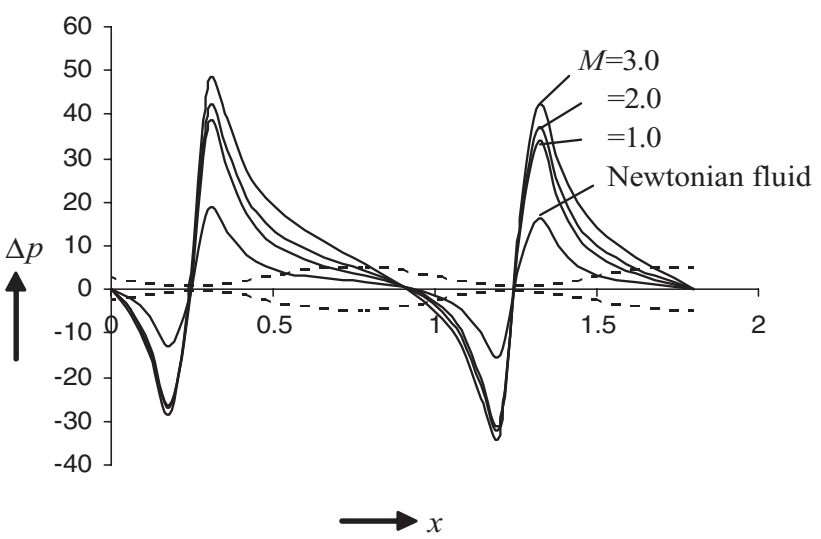

(b) $t=0.25$

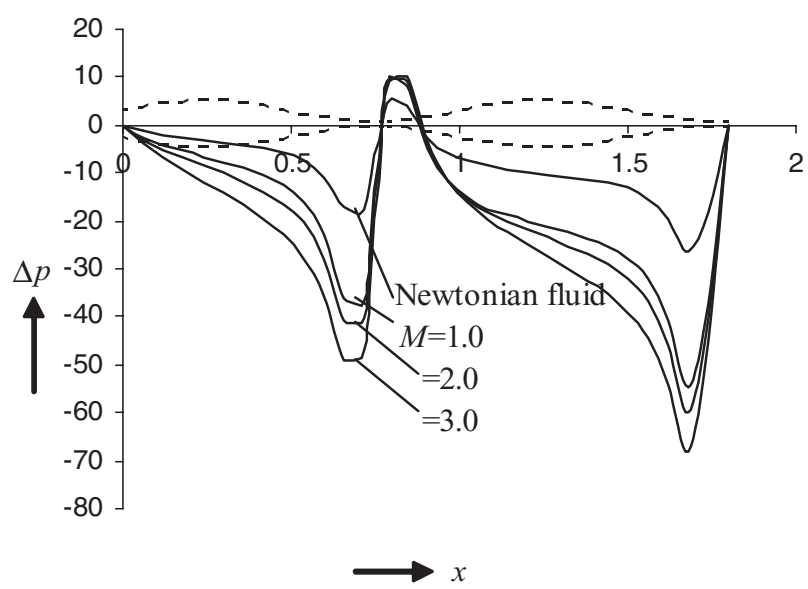

(d) $t=0.75$

Figure 3. Pressure vs. axial distance. Dotted lines represent the position of bolus and solid lines show the pressure distribution, based on Equation (10), across the length of the tube passing the Newtonian and MHD fluids for different Hartmann numbers $(M=1.0,2.0,3.0)$, $\phi=0.9$ and $l=1.8$.

are similar. The most significant difference is that the peaks of the pressure are identical in the integral case and different in the non-integral case. Furthermore, Figure 4 shows that at all temporal positions, the local wall shear stress increases with $M$.

Moreover the efficiency increases with $M$, and hence also with $B_{0}$ and $\sigma$, to maintain the same flow rate. It may be physically interpreted that the fluid requires a better pump to maintain the same flow rate under an increased magnetic field and electrical conductivity (cf. Figure 5). Precisely, the flow rate decreases with increasing magnetic field and electrical conductivity. This endorses the findings given above.

Figure 6 shows that the averaged flow rate decreases linearly with increasing pressure $(\Delta p \neq 0)$. The flow rate can be checked by applying a particular pressure at the outlet. This is true for all fluids. When $M$ is increased, the gradient of the linear relation diminishes. There is a particular point, say a critical value of pressure and flow rate, which does not change with $M$. Above the critical value, the pressure required to check the flow rate is greater for increasing $M$ and below that less pressure can check a higher flow rate. Obviously, $B_{0}$ and $\sigma$ have similar effects on the flow. This is similar to the observation of Ali et al. (2008) for MHD fluids with variable viscosity in an infinite channel.

Restrictions on the tube walls to move only in one direction play a vital role in bringing about quantitative changes (cf. Misra and Pandey 2001).

Finally, the reflux region increases very slightly for an MHD fluid when $M$ is increased (cf. Figure 7). The insignificant change is also due to the small value of $M$ that has been considered in the analysis. 


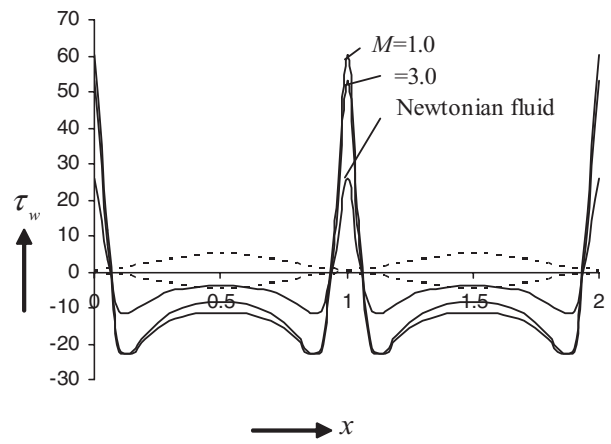

(a) $t=0.0($ similar is the distribution for $t=1.0$ )

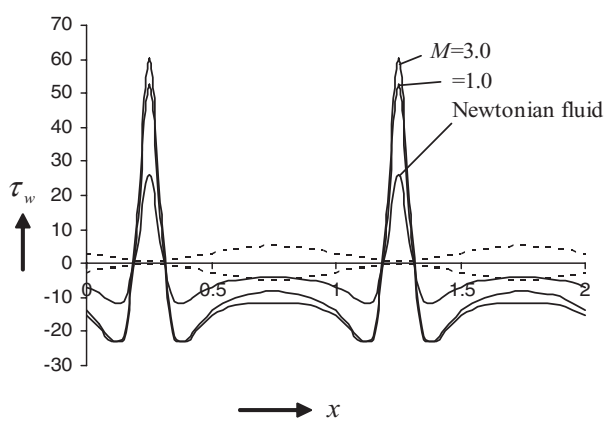

(b) $t=0.25$



(c) $t=0.5$

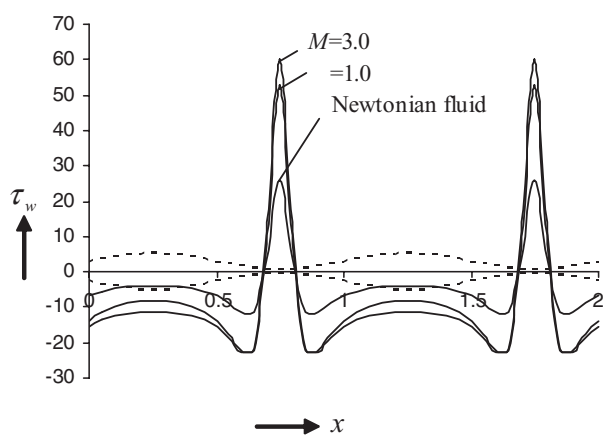

(d) $t=0.75$

Figure 4. Local wall shear stress vs. axial distance along the finite-length tube at five time instants. Dotted lines represent the position of wave, whereas solid lines represent the local wall shear stress distribution, based on Equation (18), for the Newtonian fluid and different Hartmann numbers $(M=1.0,3.0), \phi=0.9$ and $l=2.0$.

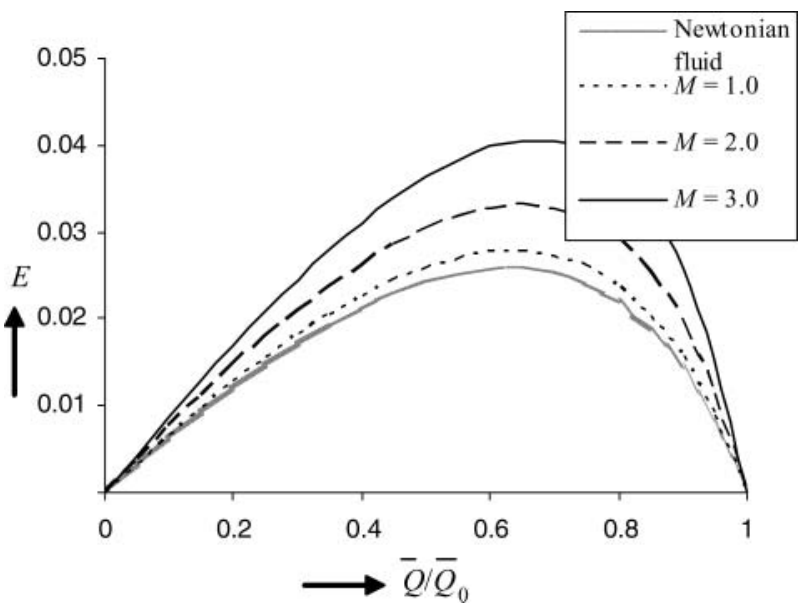

Figure 5. Mechanical efficiency vs. ratio of the averaged flow rate and maximum averaged flow rate, different lines represent efficiency of peristaltic pump, based on Equation (19), for corresponding different Hartmann numbers $(M=1.0,2.0,3.0)$ and $\phi=0.4$.

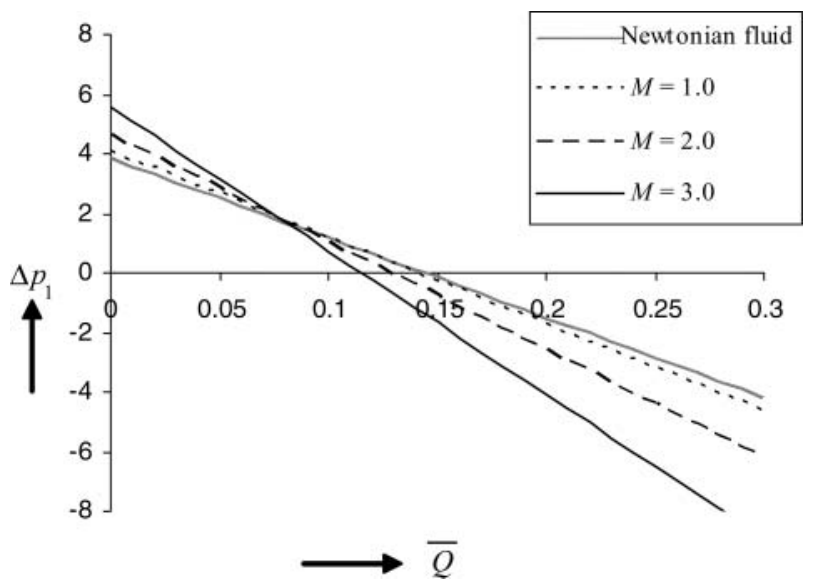

Figure 6. Pressure difference across one wavelength vs. averaged flow rate, different lines represent pressure, based on Equation (20), for corresponding different Hartmann numbers $(M=1.0,2.0,3.0)$, and $\phi=0.4$.

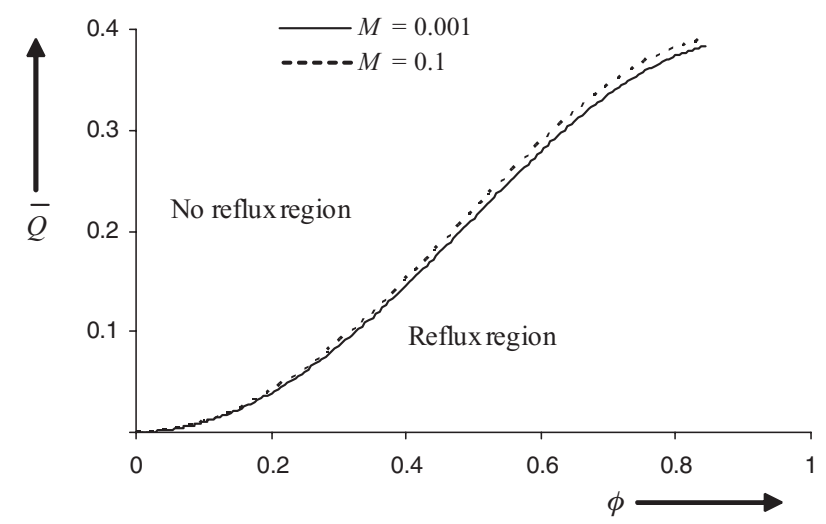

Figure 7. Averaged flow rate vs. amplitude, the different lines represent reflux limit, based on Equation (32), for corresponding Hartmann numbers $(M=0.001,0.1)$. 


\section{Conclusion}

On the basis of the above discussion, if $M=\sqrt{(\sigma / \mu)} a B_{0}$, and the transverse magnetic field, $B_{0}$, and the electric conductivity, $\sigma$, increase, the pumping machinery has to exert more pressure for pushing the fluid forward. In other words, pumping has to work more efficiently. Thus, the MHD fluid requires more effort to be pumped in comparison to Newtonian fluids. Therefore, the swallowing of saline water in the oesophagus requires more effort than that of water. Also, the local wall shear stress increases with the Hartmann number.

Furthermore, the gradient of the linear relation between the pressure for $\Delta p \neq 0$ diminishes when $M$ is increased. Critical values of pressure and flow rate do not change with $M$. The pressure required to check the flow rate above the critical value is greater for increasing $M$ and less below the critical value. Obviously, $B_{0}$ and $\sigma$ have similar effects on the flow.

It is also inferred that MHD parameters make the fluid more prone to flow reversal and so saline water is more likely to flow in the retrograde direction near the wall than normal water under similar circumstances.

\section{References}

Ali N, Hussain Q, Hayat T, Asghar S. 2008. Slip effects on the peristaltic transport of MHD fluid with variable viscosity. Phys Lett. A 372:1477-1489.

Ebaid A. 2008. Effects of magnetic field and wall slip conditions on the peristaltic transport of a Newtonian fluid in an asymmetric channel. Phys Lett. A 372:4493-4499.

El-Shehawey FE, Husseny SZA. 2002. Peristaltic transport of a magneto-fluid with porous boundaries. Appl Math Comput. 129:421-440.

Hayat T, Ali N. 2006. Peristaltic induced motion of a MHD third grade fluid in a deformable tube. Physica A 370: 225-239.

Hayat T, Ali N. 2007. A mathematical description of peristaltic hydromagnetic flow in a tube. Appl Math Comput. 188:14911502.

Hayat T, Afsar A, Khan M, Asghar S. 2007a. Peristaltic transport of a third order fluid under the effect of a magnetic field. Comput Math Appl. 53:1074-1084.
Hayat T, Ahmad N, Ali N. 2008a. Effects of endoscope and magnetic field on the peristalsis involving Jeffrey fluid. Commun Nonlinear Sci Numer Simul. 13:1581-1591.

Hayat T, Ali N. 2008. Peristaltic motion of a Jeffrey fluid under the effect of a magnetic field in a tube. Commun Nonlinear Sci Numer Simul. 13:1343-1352.

Hayat T, Javed M, Asghar S. 2008b. MHD peristaltic motion of Johnson-Segalman fluid in a channel with complaint walls. Phys Lett. A 372:5026-5036.

Hayat T, Khan AM, Siddiqui AM, Asghar S. 2007b. Non-linear peristaltic flow of a non-Newtonian fluid under effect of a magnetic field in a planner channel. Commun Nonlinear Sci Numer Simul. 12:910-919.

Kothandapani M, Srinivas S. 2008a. Peristaltic transport of a Jeffrey fluid under the effect of magnetic field in an asymmetric channel. Int J Non-linear Mech. 43:915-924.

Kothandapani M, Srinivas S. 2008b. On the influence of wall properties in the MHD peristaltic transport and the porous medium. Phys Lett. A 372:4586-4591.

Li M, Brasseur JG. 1993. Non-steady peristaltic transport in finite length tubes. J Fluid Mech. 248:129-151.

Mekheimer KhS, Abd-elmaboud Y. 2008. The influence of heat transfer and magnetic field on peristaltic transport of a Newtonian fluid in vertical annulus: application of an endoscope. Phys Lett. A 372:1657-1665.

Mekheimer KhS. 2004. Peristaltic flow of blood under the effect of a magnetic field in a non-uniform channels. Appl Math Comput. 153:763-777.

Mekheimer KhS. 2008. Effect of the induced magnetic field on the peristaltic flow of a couple stress fluid. Phys Lett. A 372:42714278.

Misra JC, Pandey SK. 2001. A mathematical model for oesophageal swallowing of a food bolus. Math Comput Model. 33:997-1009.

Naby AEHAE, Misery AEME, Kareem MFAE. 2006a. Effects of a magnetic field on trapping through peristaltic motion for generalized Newtonian fluid in channel. Physica A 367:7992.

Naby AEHAE, Misery AEME, Shamy IE. 2006b. Hydromagnetic flow of generalized Newtonian fluid through a uniform tube with peristalsis. Appl Math Comput. 173:856-871.

Shapiro AH, Jafferin MY, Weinberg SL. 1969. Peristaltic pumping with long wavelengths at low Reynolds number. J Fluid Mech. 35:669-675.

Wang Y, Hayat T, Ali N, Oberlack M. 2008. Magnetohydrodynamic peristaltic motion of a Sisko fluid in a symmetric or asymmetric channel. Physica A 387:347-362. 

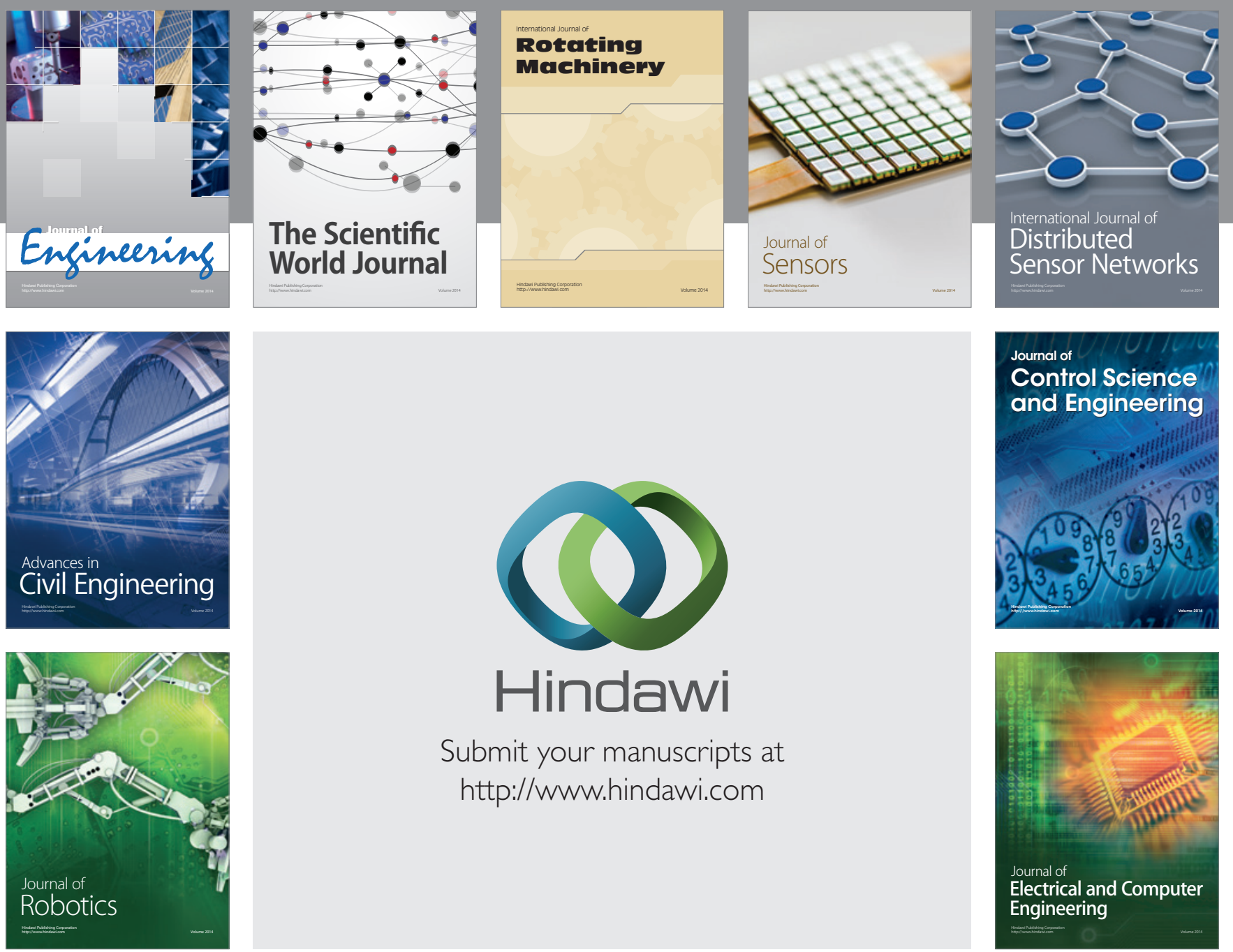

Submit your manuscripts at

http://www.hindawi.com
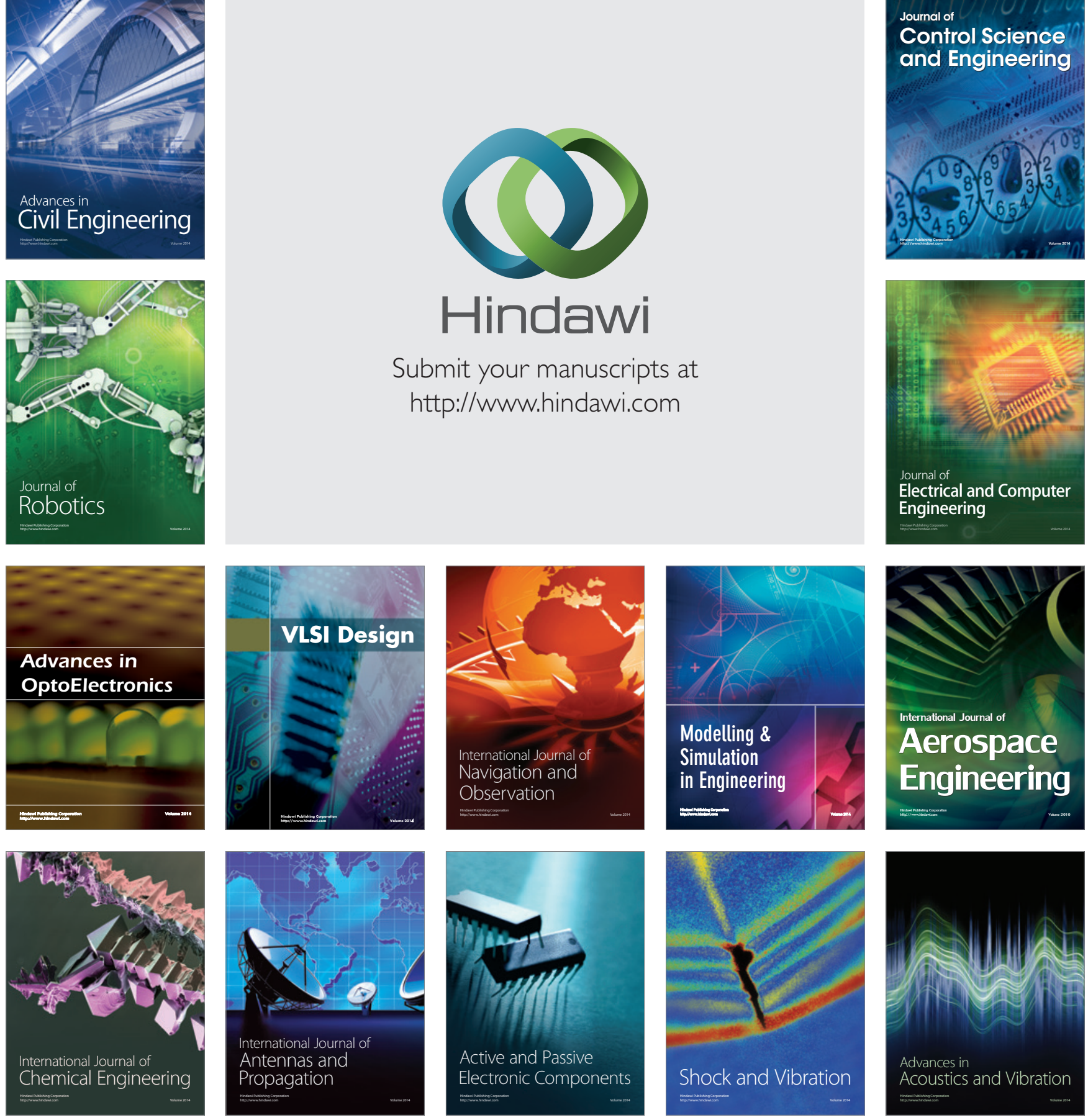